\title{
Highly Diastereo- and Enantioselective Reagents for Aldehyde Crotylation
}

\author{
Blaine M. Hackman, Pamela J. Lombardi, and James L. Leighton* \\ Department of Chemistry, Columbia University, New York, New York, 10027
}

\section{Supporting Information}

General Information. All reactions were carried out under an atmosphere of nitrogen in flame- or oven-dried glassware with magnetic stirring unless otherwise indicated. All reaction solvents were purified by degassing with argon and passage through an activated alumina column. Z- and Ecrotyltrichlorosilane were prepared according to literature procedures. ${ }^{1}{ }^{1} \mathrm{H}$ NMR spectra were recorded on Bruker DPX-300 (300 MHz) or DPX-400 (400 MHz) spectrometers. ${ }^{1} \mathrm{H}$ NMR chemical shifts $(\square)$ are reported in parts per million (ppm) relative to residual protiated solvent $\left(\mathrm{CDCl}_{3}, 7.25 ; \mathrm{C}_{6} \mathrm{D}_{6}, 7.15\right)$. Data are reported as follows: $(\mathrm{s}=$ singlet, $\mathrm{d}=$ doublet, $\mathrm{t}=$ triplet, $\mathrm{q}=$ quartet, quint $=$ quintet, $\mathrm{m}=$ multiplet, $\mathrm{dt}$ $=$ doublet of triplets, $\mathrm{dd}=$ doublet of doublets, $\mathrm{ddd}=$ doublet of doublet of doublets; coupling constant(s) in $\mathrm{Hz}$; integration; assignment). Proton decoupled ${ }^{13} \mathrm{C}$ NMR spectra were recorded on a Bruker DPX-300 $(75 \mathrm{MHz})$ and are reported in ppm from $\mathrm{CDCl}_{3}$ internal standard $(77.0 \mathrm{ppm}) .{ }^{13} \mathrm{C}$ resonances are singlets, unless otherwise specified. ${ }^{31}$ P NMR spectra were recorded on a Bruker DPX-300 (121.6 MHz) and are reported in ppm from $\mathrm{H}_{3} \mathrm{PO}_{4}\left(85 \%\right.$ in $\left.\mathrm{H}_{2} \mathrm{O}\right)$ external standard $(0.0 \mathrm{ppm}) .{ }^{19} \mathrm{~F}$ NMR spectra were recorded on a Bruker DPX-300 (282 MHz) and are reported in ppm from internal $\square, \square, \square$-trifluorotoluene $(0.00$ ppm). Infrared spectra were recorded on a Perkin Elmer Paragon 1000 FT-IR spectrometer. Optical rotations were recorded on a JASCO DIP-1000 digital polarimeter; the concentration $c$ is reported in $\mathrm{g} / 100 \mathrm{~mL}$. Low resolution mass spectra were obtained on a JEOL HX110 mass spectrometer in the Columbia University Mass Spectrometry Laboratory.

(1) (a) Tsuji, J.; Hara, M.; Ohno, K. Tetrahedron 1974, 30, 2143-2146. (b) Furuya, N.; Sukawa, T. J. Organomet. Chem. 1975, 96, C1-C3. (c) Kira, M.; Hino, T.; Sakurai, H. Tetrahedron Lett. 1989, 30, 1099-1102. (d) Iseki, K.; Kuroki, Y.; Takahashi, M.; Kishimoto, S.; Kobayashi, Y. Tetrahedron 1997, 53, 3513-3526. 
Preparation of reagent $(\boldsymbol{R}, \boldsymbol{R})-2$ : (Note: Reagents 2 and $\mathbf{3}$ are moisture sensitive, and it is important that care be taken to exclude moisture especially during the workup procedure. The filtrations and crystallization are most effectively carried out in an inert atmosphere glovebox, especially in humid environments.) To a cooled $\left(0^{\circ} \mathrm{C}\right)$ solution of $Z$-crotyltrichlorosilane $(8.38 \mathrm{~mL}, 0.055 \mathrm{~mol})$ in $\mathrm{CH}_{2} \mathrm{Cl}_{2}$ (100 mL) was added 1,8-diazobicyclo[5.4.0]undec-7-ene (DBU) (16.45g, $0.110 \mathrm{~mol})$. A solution of $(R, R)$ $N, N$ '-bis-(4-bromobenzyl)cyclohexane-1,2-diamine' $22.56 \mathrm{~g}, 0.050 \mathrm{~mol})$ in $\mathrm{CH}_{2} \mathrm{Cl}_{2}(50 \mathrm{~mL})$ was then added over $30 \mathrm{~min}$. The reaction mixture was allowed to warm to room temperature. After $12 \mathrm{~h}$ the reaction mixture was concentrated by vacuum distillation (the use of a rotary evaporator is not recommended, as substantial decomposition can result). The residue was diluted with pentane $(150 \mathrm{~mL})$ and vigorously stirred for $3 \mathrm{~h}$ to ensure complete precipitation of all DBU salts. The suspension was filtered and the filtrate was placed in a freezer $\left(-10^{\circ} \mathrm{C}\right)$ for two days. The resulting pale yellow solid was isolated by filtration and dried under vacuum to give $(R, R)-2(21.5 \mathrm{~g}, 76 \%$ yield $)$. [ $\square]_{\mathrm{D}}=-46.1^{\circ}(c 1.46$, $\left.\mathrm{CH}_{2} \mathrm{Cl}_{2}\right) ;{ }^{1} \mathrm{H}$ NMR (300 MHz, $\left.\mathrm{C}_{6} \mathrm{D}_{6}\right) \square$ 7.38-7.35 (m, 4H, Ar-H), 7.14-7.10 (m, 4H, Ar-H), 5.49-5.36 (m, $\left.2 \mathrm{H}, \mathrm{SiCH}_{2} \mathrm{CH}=\mathrm{CHMe}\right), 3.99-3.92\left(\mathrm{~m}, 2 \mathrm{H}\right.$, two of $\left.\operatorname{ArCH}_{2} \mathrm{~N}\right), 3.64-3.52\left(\mathrm{~m}, 2 \mathrm{H}\right.$, two of $\left.\operatorname{ArCH}_{2} \mathrm{~N}\right), 2.72-$ 2.61 (m, 2H, two CHN), 1.79-1.31 (m, 9H, $\mathrm{SiCH}_{2}, \mathrm{CH}=\mathrm{CHCH}_{3}$, and 2 x $\mathrm{CH}_{2}$ ), 0.96-0.84 (m, 4H, 2 x $\left.\mathrm{CH}_{2}\right) ;{ }^{13} \mathrm{C}$ NMR $\left(75 \mathrm{MHz}, \mathrm{CDCl}_{3}\right) \square 141.6,140.7,131.6,130.2,129.3,125.3,122.3,66.6,65.8,47.5$, 31.1, 30.8, 25.1, 25.0, 18.9, 13.4; ${ }^{29} \mathrm{Si} \mathrm{NMR}\left(60 \mathrm{MHz}, \mathrm{CDCl}_{3}\right) \square-4.0$; IR $\left(\mathrm{CH}_{2} \mathrm{Cl}_{2}\right)$ 3020, 2940, 2863, $1487,1444,1287,1207,1175,1106,1070,1012 \mathrm{~cm}^{-1}$.

Preparation of reagent $(\boldsymbol{R}, \boldsymbol{R})-3$ : Using $E$-crotyltrichlorosilane, the procedure is analogous to that for $(\boldsymbol{R}, \boldsymbol{R})-2$ with the following modification: After filtration of the DBU salts, $15 \mathrm{~mL} \mathrm{CH}_{2} \mathrm{Cl}_{2}$ was added to the filtrate. The transparent, pale yellow solution was then placed in a freezer $\left(-10^{\circ} \mathrm{C}\right)$ for 2 days. The resulting pale white crystals were isolated by filtration and dried under vacuum to give $(R, R)-3(4.92 \mathrm{~g}$, $69 \%$ yield; a larger scale run gave $28.1 \mathrm{~g}, 62 \%$ yield $)$. [ $]_{\mathrm{D}}=-43.5^{\circ}\left(\mathrm{c} 1.25, \mathrm{CHCl}_{3}\right) ;{ }^{1} \mathrm{H} \mathrm{NMR}(300 \mathrm{MHz}$, $\left.\mathrm{CDCl}_{3}\right) \square$ 7.45-7.42 (m, 4H, Ar-H), 7.24-7.20 (m, 4H, Ar-H), 5.31-5.24 (m, 2H, $\left.\mathrm{R}_{3} \mathrm{SiCH}_{2} \mathrm{CH}=\mathrm{CHMe}\right)$, $3.97\left(\mathrm{AB} \mathrm{q}, 2 \mathrm{H}, J=16.2 \mathrm{~Hz}, \operatorname{ArCH}_{2} \mathrm{~N}\right), 3.92\left(\mathrm{AB} \mathrm{q}, 2 \mathrm{H}, J=15.1 \mathrm{~Hz}, \operatorname{ArCH}_{2} \mathrm{~N}\right), 2.78-2.71(\mathrm{~m}, 2 \mathrm{H}$, two 
$\mathrm{CHN}), 1.81-1.53\left(\mathrm{~m}, 9 \mathrm{H}, \mathrm{SiCH}_{2}, \mathrm{CH}=\mathrm{CHCH}_{3}\right.$, and $\left.2 \times \mathrm{CH}_{2}\right), 1.15-0.92\left(\mathrm{~m}, 4 \mathrm{H}, 2 \times \mathrm{CH}_{2}\right) ;{ }^{13} \mathrm{C} \mathrm{NMR}(75$ $\left.\mathrm{MHz}, \mathrm{CDCl}_{3}\right) \square 141.5,140.8,131.5,130.2,129.4,127.3,123.1$, 66.5, 65.9, 47.5, 31.0, 30.8, 25.1, 25.0, 23.5, 18.4; $\left.{ }^{29} \mathrm{Si} \mathrm{NMR} \mathrm{(60} \mathrm{MHz,} \mathrm{CDCl}_{3}\right) \square-4.2 ; \mathrm{IR}\left(\mathrm{CH}_{2} \mathrm{Cl}_{2}\right)$ 3019, 2939, 2862, 1592, 1487, 1175,1106 $\mathrm{cm}^{-1}$.

General Procedure for Aldehyde Crotylation Reactions Using $(\boldsymbol{R}, \boldsymbol{R})-2$ and $(\boldsymbol{R}, \boldsymbol{R})-3$ : To a cooled $\left(0{ }^{\circ} \mathrm{C}\right)$ solution of reagent 2 or reagent $3(0.707 \mathrm{~g}, 1.26 \mathrm{mmol})$ in $\mathrm{CH}_{2} \mathrm{Cl}_{2}(12 \mathrm{~mL})$ is added the aldehyde $(1.14 \mathrm{mmol})$. After $20 \mathrm{~h}$ the reaction is quenched by the addition of $4 \mathrm{~mL}$ of a $1 \mathrm{M} \mathrm{HCl} \mathrm{solution}$, and the resulting mixture is stirred for $10 \mathrm{~min}$. The layers are separated and the aqueous layer is extracted with ethyl acetate $(2 \times 10 \mathrm{~mL})$. The combined organic layers are dried over $\mathrm{MgSO}_{4}$, filtered, and concentrated. Purification of the residue by silica gel chromatography provides the pure homoallylic alcohol products.

Procedure for the Larger Scale Crotylation of Hydrocinnamaldehyde Using Reagent 2 with Recovery of the Diamnie: To a cooled $\left(0^{\circ} \mathrm{C}\right)$ solution of reagent $2(9.77 \mathrm{~g}, 17.1 \mathrm{mmol})$ in $150 \mathrm{~mL}$ was added hydrocinnamaldehyde $(2.06 \mathrm{~mL}, 15.6 \mathrm{mmol})$. After $20 \mathrm{~h}$ the reaction was quenched with $100 \mathrm{~mL}$ of saturated aqueous $\mathrm{NaHCO}_{3}$, and the mixture was stirred for $10 \mathrm{~min}$. The layers were separated and the aqueous layer was extracted with ethyl acetate $(2 \times 50 \mathrm{~mL})$. The combined organic layers were dried over $\mathrm{MgSO}_{4}$, filtered, and concentrated. Purification of the residue by chromatography on silica gel (10\% EtOAc:90\% hexanes) afforded $2.34 \mathrm{~g}(79 \%)$ of $(3 R, 4 R)-4-$ methyl-1-phenyl-5-hexen-3-ol as an oil. The column was then eluted with hexanes/EtOAc/Et $3 \mathrm{~N}$ (1:1:0.1) to provide $6.95 \mathrm{~g}$ (90\%) of diamine 1.

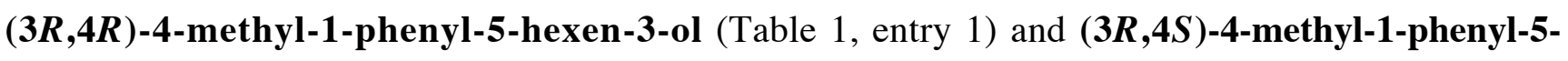
hexen-3-ol (Table 2, entry 1): These compounds are known. ${ }^{3}$ Enantiomeric excess was determined by chiral HPLC: Chiracel OD column; 97:3 hexane:isopropanol; $1.0 \mathrm{~mL} / \mathrm{min}, 254 \mathrm{~nm}$. The assay was developed using a 2.5:1 syn:anti mixture of racemic diastereomers.

(3) Kobayashi, S.; Nishio, K. J. Org. Chem. 1994, 59, 6620-6628. 

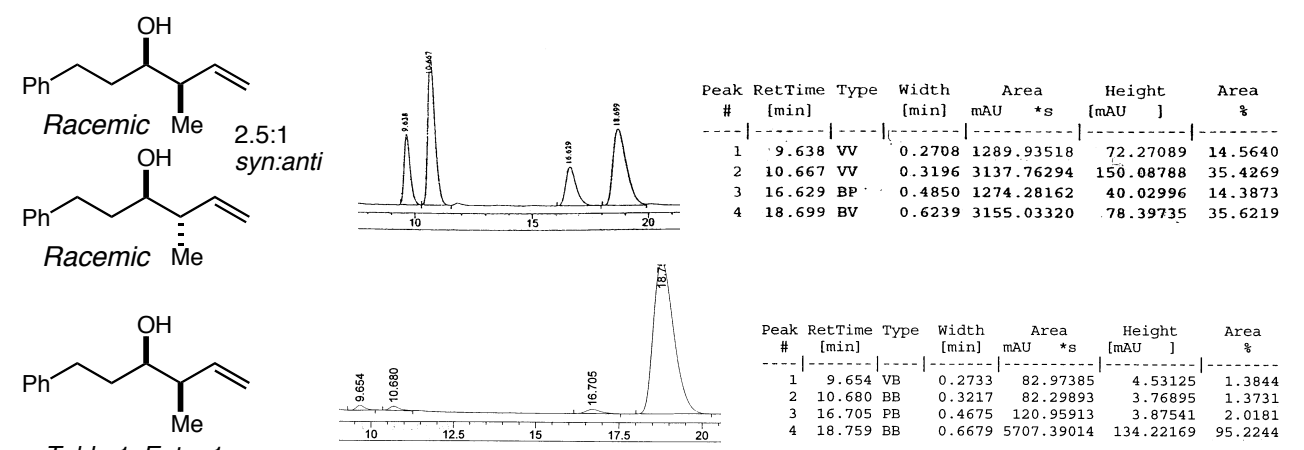

Table 1, Entry 1
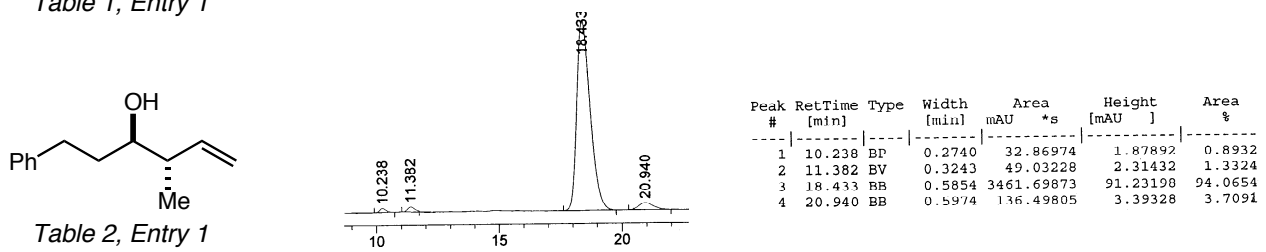

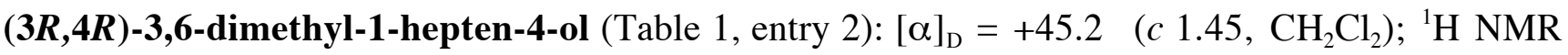
$\left(300 \mathrm{MHz}, \mathrm{CDCl}_{3}\right) \square$ 5.85-5.74 $\left(\mathrm{m}, 1 \mathrm{H}, \mathrm{CH}=\mathrm{CH}_{2}\right), 5.13-5.05\left(\mathrm{~m}, 2 \mathrm{H}, \mathrm{CH}=\mathrm{CH}_{2}\right), 3.61-3.58(\mathrm{~m}, 1 \mathrm{H}$, $\mathrm{CHOH}), 2.32-2.21\left(\mathrm{~m}, 1 \mathrm{H}, \mathrm{CHCH}_{3}\right), 1.87-1.74\left(\mathrm{~m}, 1 \mathrm{H},\left(\mathrm{CH}_{3}\right)_{2} \mathrm{CH}\right), 1.45(\mathrm{~s}, 1 \mathrm{H}, \mathrm{OH}), 1.40-1.22(\mathrm{~m}, 2 \mathrm{H}$, $\left.\left(\mathrm{CH}_{3}\right)_{2} \mathrm{CHCH}_{2}\right), 1.05\left(\mathrm{~d}, 3 \mathrm{H}, J=6.9 \mathrm{~Hz}, \mathrm{CHCH}_{3}\right), 0.95\left(\mathrm{~d}, 3 \mathrm{H}, J=6.6 \mathrm{~Hz}\right.$, one of $\left.\left(\mathrm{CH}_{3}\right)_{2} \mathrm{CH}\right), 0.92(\mathrm{~d}, 3 \mathrm{H}$, $J=6.6 \mathrm{~Hz}$, one of $\left.\left(\mathrm{CH}_{3}\right)_{2} \mathrm{CH}\right) ;{ }^{13} \mathrm{C} \mathrm{NMR}\left(75 \mathrm{MHz}, \mathrm{CDCl}_{3}\right) \square 141.4,115.7,72.9,44.2,43.4,25.1,24.2$, 22.1, 14.5; IR $\left(\mathrm{CH}_{2} \mathrm{Cl}_{2}\right)$ 3368, 3082, 2959, 1638, 1461, 1373, 1259, 1088, $912 \mathrm{~cm}^{-1}$.

Enantiomeric excess was determined by chiral HPLC analysis of the 3,5-dinitrobenzoate ester: Chiracel AD-H column; 98.8:0.6:0.6 hexane:EtOH:MeOH; $1.0 \mathrm{~mL} / \mathrm{min}, 254 \mathrm{~nm}$.
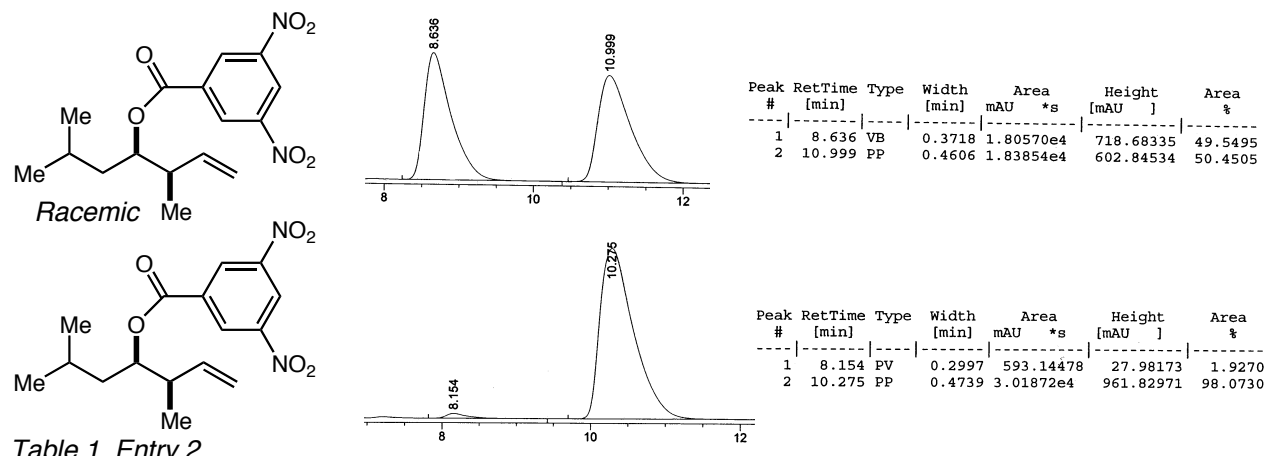

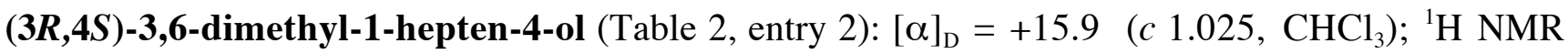
$\left(300 \mathrm{MHz}, \mathrm{CDCl}_{3}\right) \square$ 5.82-5.70 (m, $\left.1 \mathrm{H}, \mathrm{CH}=\mathrm{CH}_{2}\right), 5.17-5.08\left(\mathrm{~m}, 2 \mathrm{H}, \mathrm{CH}=\mathrm{CH}_{2}\right), 3.51-3.45(\mathrm{~m}, 1 \mathrm{H}$, $\mathrm{CHOH}), 2.22-2.15\left(\mathrm{~m}, 1 \mathrm{H}, \mathrm{CHCH}_{3}\right), 1.86-1.81\left(\mathrm{~m}, 1 \mathrm{H},\left(\mathrm{CH}_{3}\right)_{2} \mathrm{CH}\right), 1.52(\mathrm{~s}, 1 \mathrm{H}, \mathrm{OH}), 1.39-1.22(\mathrm{~m}, 2 \mathrm{H}$, $\left.\left(\mathrm{CH}_{3}\right)_{2} \mathrm{CHCH}_{2}\right), 1.04\left(\mathrm{~d}, 3 \mathrm{H}, J=6.9 \mathrm{~Hz}, \mathrm{CHCH}_{3}\right), 0.94\left(\mathrm{~d}, 3 \mathrm{H}, J=6.8 \mathrm{~Hz}\right.$, one of $\left.\left(\mathrm{CH}_{3}\right)_{2} \mathrm{CH}\right), 0.91(\mathrm{~d}, 3 \mathrm{H}$, 
$J=6.6 \mathrm{~Hz}$, one of the $\left.\left(\mathrm{CH}_{3}\right)_{2} \mathrm{CH}\right) ;{ }^{13} \mathrm{C} \mathrm{NMR}\left(75 \mathrm{MHz}, \mathrm{CDCl}_{3}\right) \square 140.7,116.8,73.0,45.1,43.9,25.0,24.2$, 22.1, 16.6; IR $\left(\mathrm{CH}_{2} \mathrm{Cl}_{2}\right) 3373,2964,2919,1606,1261,1093,1016 \mathrm{~cm}^{-1}$.

Enantiomeric excess was determined by chiral HPLC analysis of the 3,5-dinitrobenzoate ester: Chiracel AD-H column; 98.8:0.6:0.6 hexane:EtOH:MeOH; $1.0 \mathrm{~mL} / \mathrm{min}, 254 \mathrm{~nm}$.
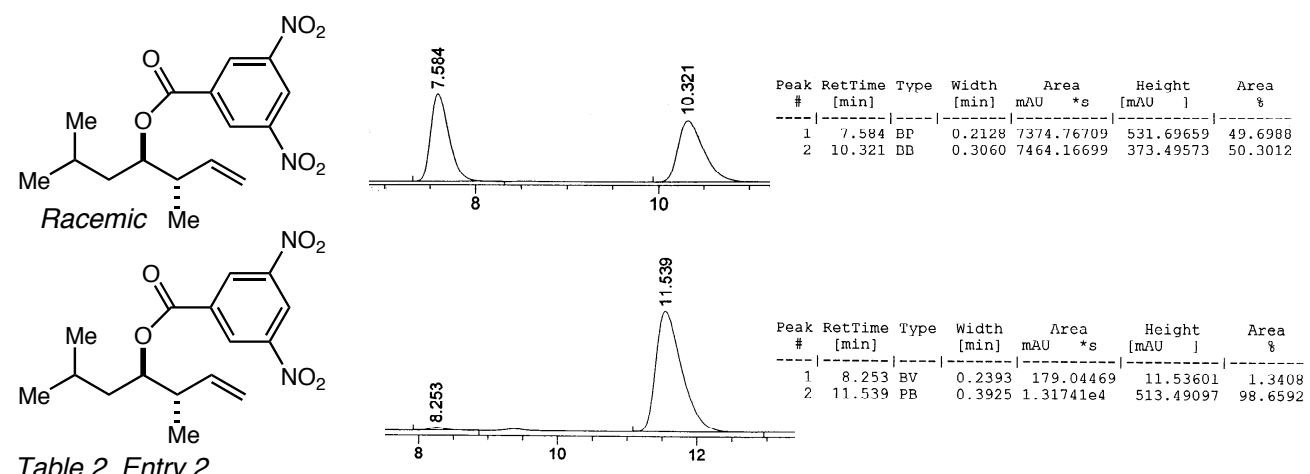

$(3 R, 4 R)-4-c y c l o h e x y l-3-m e t h y l-1-b u t e n-4-o l ~(T a b l e ~ 1, ~ E n t r y ~ 3): ~[\square]_{D}=+39.2^{\circ}\left(c 0.67, \mathrm{CHCl}_{3}\right)$. This is a known compound. ${ }^{3}$ Enantiomeric excess was determined by chiral HPLC analysis of the 3,5dinitrobenzoate ester: Chiracel AD-H column; 98:1:1 hexane:EtOH:MeOH; $1.0 \mathrm{~mL} / \mathrm{min}, 254 \mathrm{~nm}$.

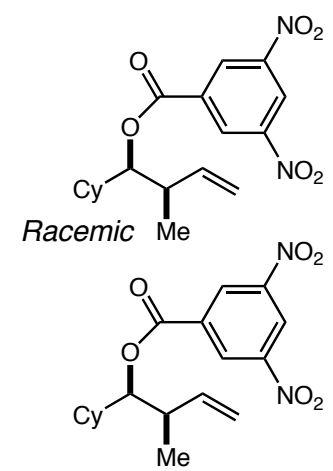

Table 1, Entry 3
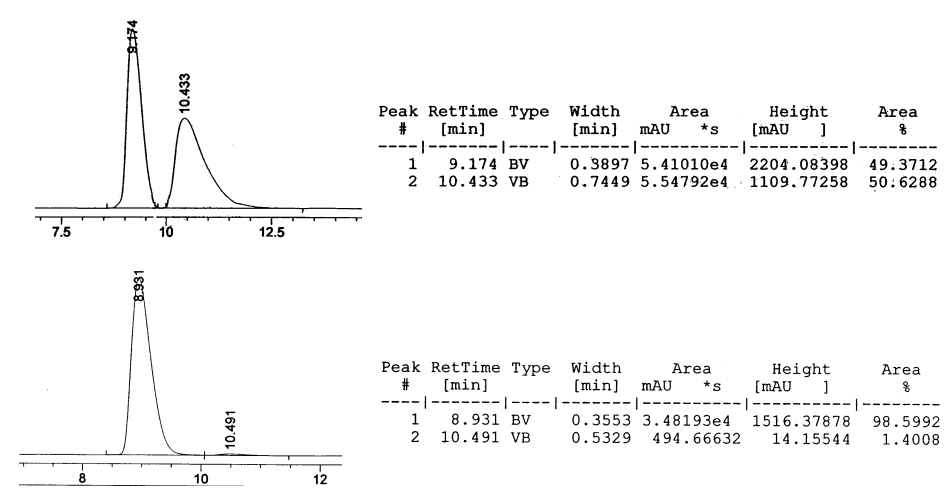

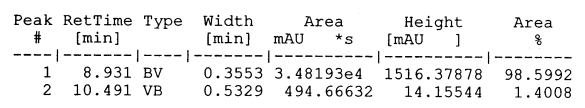

(3S,4R)-4-cyclohexyl-3-methyl-1-buten-4-ol (Table 2, Entry 3): $[\square]_{\mathrm{D}}=-16.8^{\circ}\left(c 1.10, \mathrm{CHCl}_{3}\right)$. This is a known compound. ${ }^{3}$ Enantiomeric excess was determined by chiral HPLC analysis of the 3,5dinitrobenzoate ester: Chiracel AD-H column; 98:1:1 hexane:EtOH:MeOH; $1.0 \mathrm{~mL} / \mathrm{min}, 254 \mathrm{~nm}$. 

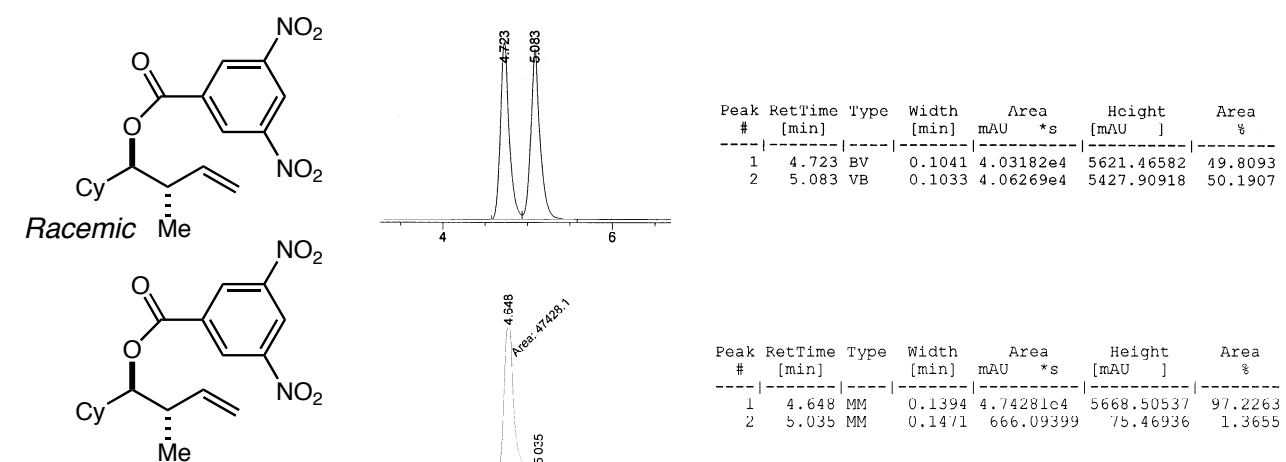

Table 2, Entry 3
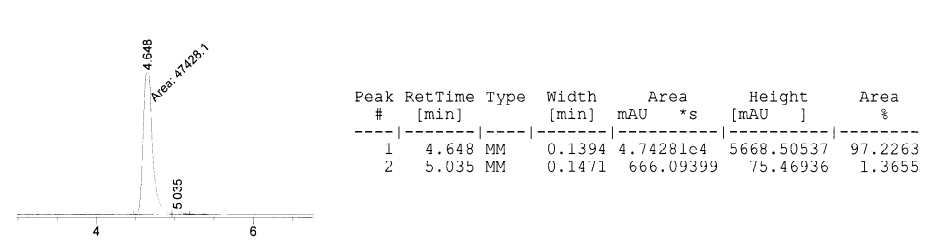

(2R,3R)-1-benzyloxy-3-methyl-4-penten-2-ol (Table 1, Entry 4): [ $]_{\mathrm{D}}=+24.6^{\circ}\left(\mathrm{c} 1.84, \mathrm{CH}_{2} \mathrm{Cl}_{2}\right)$; ${ }^{1} \mathrm{H}$ NMR (300 MHz, $\left.\mathrm{CDCl}_{3}\right) \square 7.43-7.36(\mathrm{~m}, 5 \mathrm{H}, \mathrm{Ar}-\mathbf{H}), 5.89-5.78\left(\mathrm{~m}, 1 \mathrm{H}, \mathrm{CH}=\mathrm{CH}_{2}\right)$, 5.18-5.10 (m, $2 \mathrm{H}$, $\left.\mathrm{CH}=\mathrm{CH}_{2}\right), 4.64\left(\mathrm{~s}, 2 \mathrm{H}, \mathrm{PhCH}_{2} \mathrm{O}\right), 3.78-3.72(\mathrm{~m}, 1 \mathrm{H}, \mathrm{CHOH}), 3.55(\mathrm{dd}, 1 \mathrm{H}, J=3.0$ and $9.4 \mathrm{~Hz}$, one of $\left.\mathrm{BnOCH}_{2}\right), 3.40\left(\mathrm{dd}, 1 \mathrm{H}, J=7.7\right.$ and $9.4 \mathrm{~Hz}$, one of $\left.\mathrm{BnOCH}_{2}\right), 2.50-2.40\left(\mathrm{~m}, 2 \mathrm{H}, \mathrm{OH}\right.$ and $\left.\mathrm{CH}_{3} \mathrm{CH}\right), 1.19$ $\left(\mathrm{d}, 3 \mathrm{H}, J=6.8 \mathrm{~Hz}, \mathrm{CH}_{3}\right) ;{ }^{13} \mathrm{C} \mathrm{NMR}\left(75 \mathrm{MHz}, \mathrm{CDCl}_{3}\right) \square 140.7,138.4,128.9,128.2,128.1,115.6,73.8$, 73.2, 41.5, 16.1; IR $\left(\mathrm{CH}_{2} \mathrm{Cl}_{2}\right)$ 3449, 2916, 2865, 1640, 1454, 1103, $916 \mathrm{~cm}^{-1}$; LRMS (FAB+) calc'd for $\mathrm{C}_{13} \mathrm{H}_{18} \mathrm{O}_{2}$ 206.28, found $\left(\mathrm{M}+\mathrm{H}^{+}\right)$207.24. (2R,3S)-1-benzyloxy-3-methyl-4-penten-2-ol (Table 2, Entry 4): $[\square]_{\mathrm{D}}=-5.6^{\circ}\left(\mathrm{c} 1.23, \mathrm{CHCl}_{3}\right)$. This is a known compound. ${ }^{4}$ Enantiomeric excess was determined by chiral HPLC: Chiracel AD-H column; 99:1 hexane:isopropanol; $1.0 \mathrm{~mL} / \mathrm{min}, 254 \mathrm{~nm}$. The assay was developed using a 4:1 anti:syn mixture of racemic diastereomers.

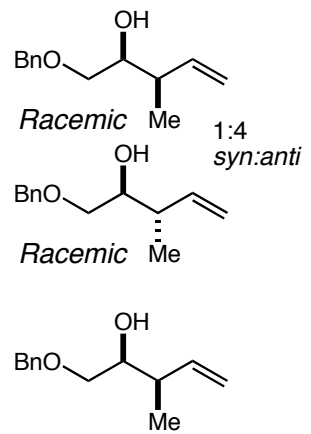

Table 1, Entry 4

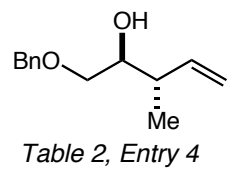

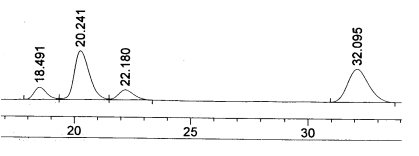
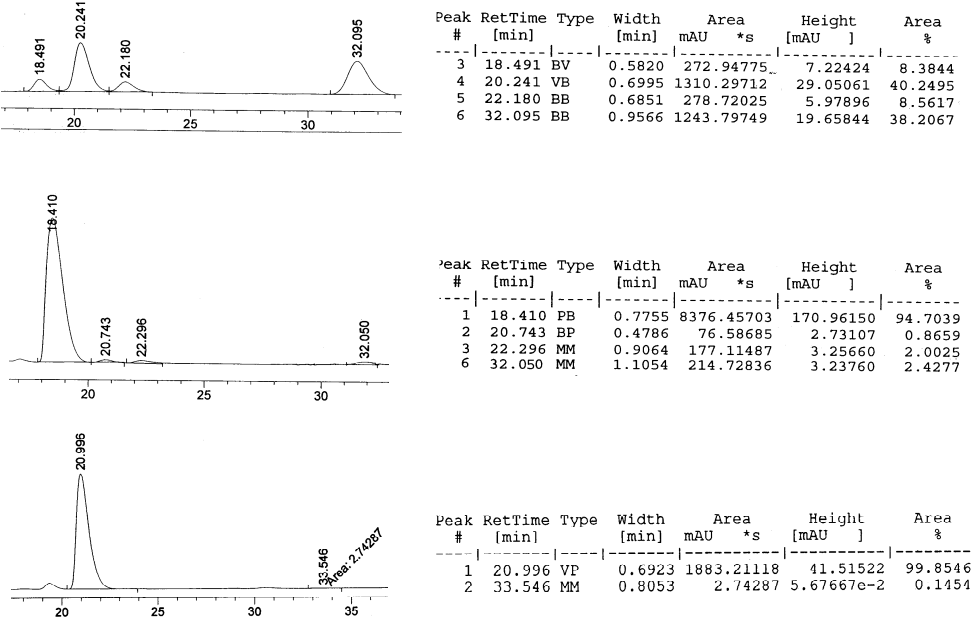

${ }^{4}$ Hoffmann, R. W.; Helbig, W. Chem. Ber. 1981, 114, 2802-2807. 


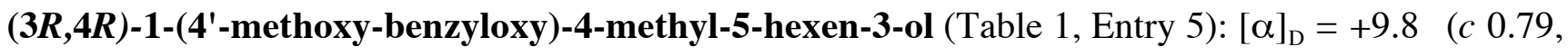
$\left.\mathrm{CH}_{2} \mathrm{Cl}_{2}\right) ;{ }^{1} \mathrm{H}$ NMR $\left(300 \mathrm{MHz}, \mathrm{C}_{6} \mathrm{D}_{6}\right) \square 7.19\left(\mathrm{~d}, 2 \mathrm{H}, J=6.5 \mathrm{~Hz}, 3^{\prime}, 5^{\prime}-\mathrm{ArH}\right), 6.81\left(\mathrm{~d}, 2 \mathrm{H}, J=6.5 \mathrm{~Hz}, 2^{\prime}, 6^{\prime}-\right.$ $\operatorname{ArH})$, 5.88-5.76 (m, 1H, CH=CH $), 5.04\left(\mathrm{t}, 2 \mathrm{H}, J=9.3 \mathrm{~Hz}, \mathrm{CH}=\mathrm{CH}_{2}\right), 3.95\left(\mathrm{~s}, 2 \mathrm{H}, \operatorname{ArCH}_{2} \mathrm{O}\right), 3.69-3.63$ $(\mathrm{m}, 1 \mathrm{H}, \mathrm{CHOH}), 3.59-3.54\left(\mathrm{~m}, 1 \mathrm{H}\right.$, one of $\left.\mathrm{ArCH}_{2} \mathrm{OCH}_{2}\right), 3.51-3.40\left(\mathrm{~m}, 1 \mathrm{H}\right.$, one of $\left.\mathrm{ArCH}_{2} \mathrm{OCH}_{2}\right), 3.35(\mathrm{~s}$, $\left.3 \mathrm{H}, \mathrm{OCH}_{3}\right), 2.75(\mathrm{~d}, 1 \mathrm{H}, J=3.4 \mathrm{~Hz}, \mathrm{OH}), 2.28\left(\mathrm{~m}, 1 \mathrm{H}, \mathrm{CHCH}_{3}\right), 1.68\left(\mathrm{~m}, 2 \mathrm{H}, \mathrm{OCH}_{2} \mathrm{CH}_{2}\right), 1.16(\mathrm{~d}, 3 \mathrm{H}, J$ $\left.=6.8 \mathrm{~Hz}, \mathrm{CH}_{3}\right) ;{ }^{13} \mathrm{C} \mathrm{NMR}\left(75 \mathrm{MHz}, \mathrm{CDCl}_{3}\right)$ 159.6, 141.4, 130.4, 129.7, 115.3, 114.2, 74.9, 73.4, 69.6, 55.6, 44.3, 33.9, 15.5; IR $\left(\mathrm{CH}_{2} \mathrm{Cl}_{2}\right)$ 3456, 2958, 2864, 1613, 1514, 1462, 1302, 1249, 1088, $1036 \mathrm{~cm}^{-1}$; LRMS (FAB+) calc'd for $\mathrm{C}_{15} \mathrm{H}_{22} \mathrm{O}_{3}$ 250.33, found $\left(\mathrm{M}+\mathrm{H}^{+}\right)$251.25. (3R, 4S)-1-(4'-methoxybenzyloxy)-4methyl-5-hexen-3-ol (Table 2, Entry 5): $[\square]_{\mathrm{D}}=-2.38^{\circ}\left(c 0.80, \mathrm{CHCl}_{3}\right)$. This is a known compound. ${ }^{5}$ Enantiomeric excess was determined by chiral HPLC analysis of the 3,5-dinitrobenzoate ester: Chiracel AD-H column; 98:1:1 hexane:EtOH:MeOH; $1.0 \mathrm{~mL} / \mathrm{min}, 254 \mathrm{~nm}$. The assay was developed using a 3:1 anti:syn mixture of racemic diastereomers.
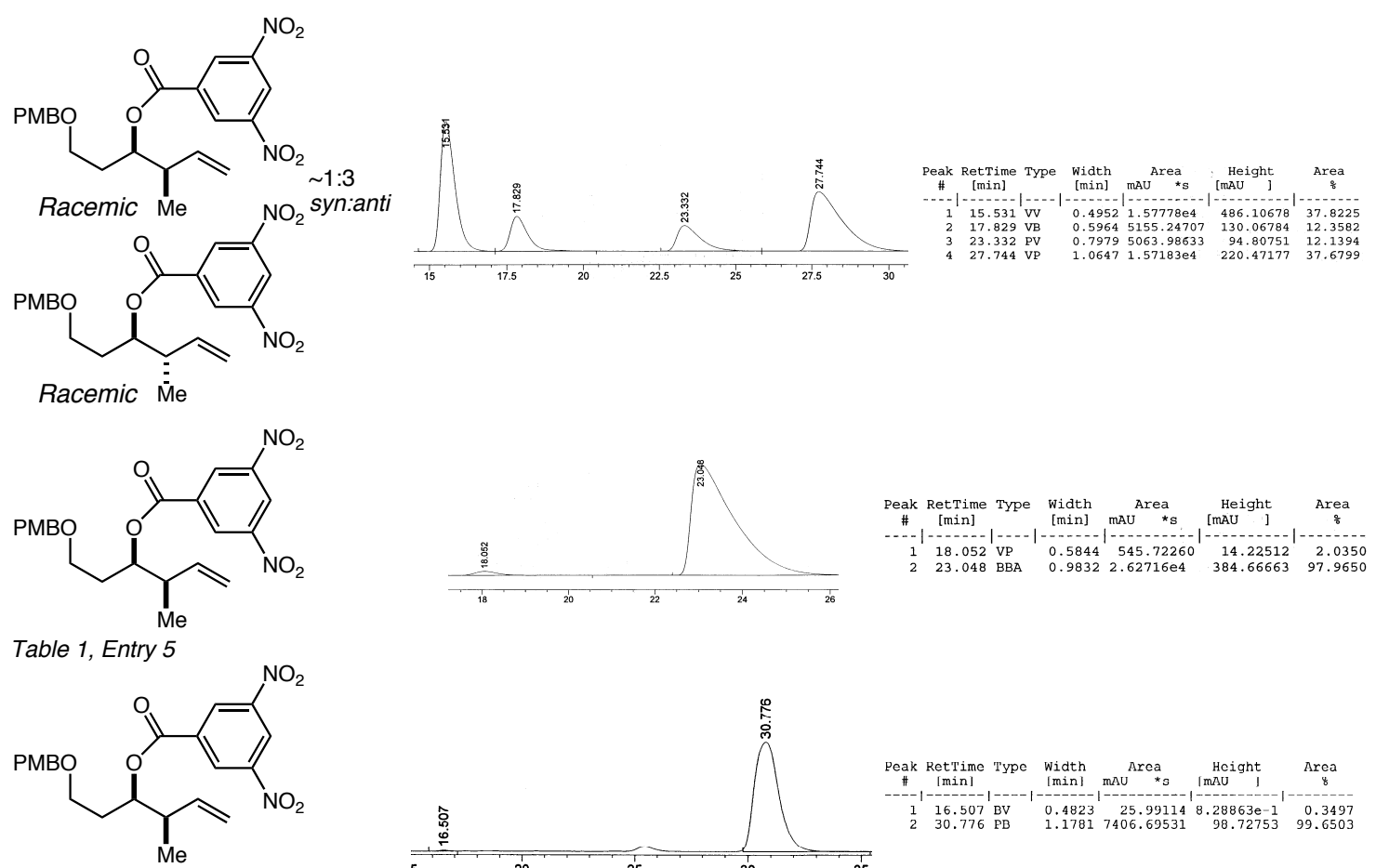

Table 2, Entry 5
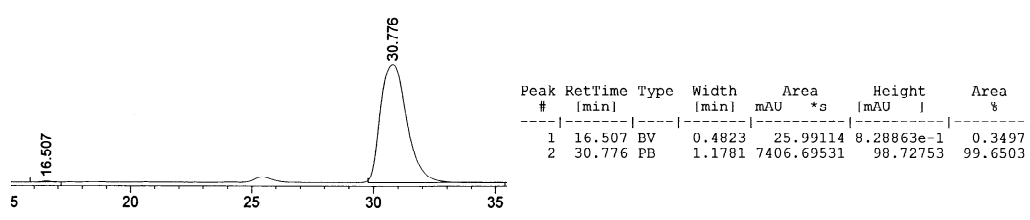

(5) Eggen, J.; Mossman, C. J.; Buck, S. B.; Nair, S. K.; Bhat, L.; Ali, S. M.; Reiff, E. A.; Boge, T. C.; Georg, G. I. J. Org. Chem. 2000, 65, 7792-7799. 
(1S,2R)-2-methyl-1-phenyl-3-buten-1-ol (Table 1, Entry 6): $[\square]_{\mathrm{D}}=-25.9^{\circ}\left(c 0.805, \mathrm{CHCl}_{3}\right)$. This is a known compound. ${ }^{6}$ Enantiomeric excess was determined by chiral HPLC analysis of the 3,5dinitrobenzoate ester: Chiracel AD-H column; 95:5 hexane:isopropanol; $1.0 \mathrm{~mL} / \mathrm{min}, 254 \mathrm{~nm}$.
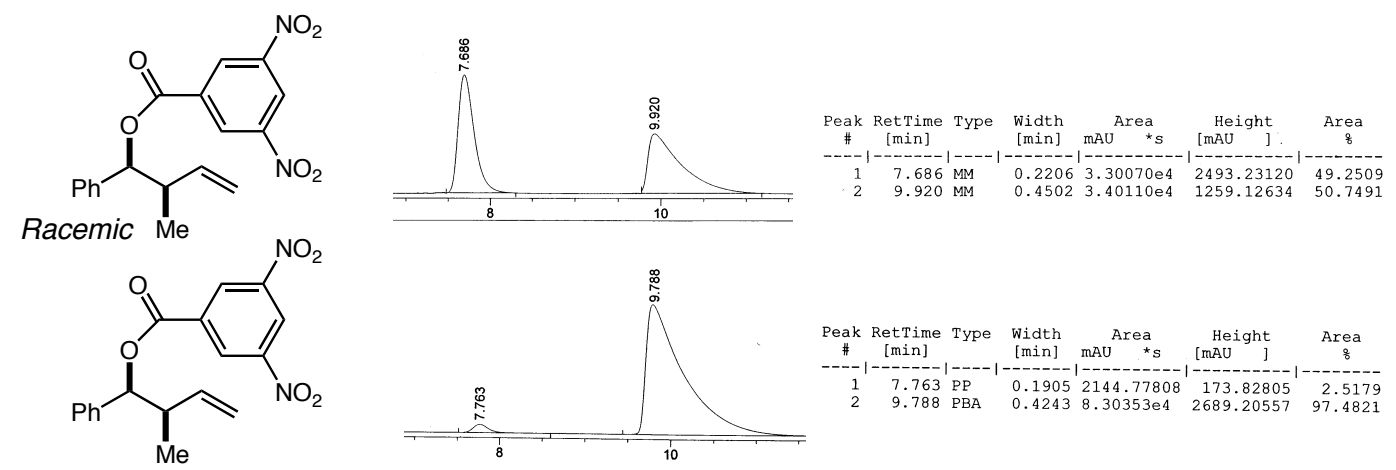

Table 1, Entry 6

(1S,2S)-2-methyl-1-phenyl-3-buten-1-ol (Table 2, Entry 6): $[\square]_{\mathrm{D}}=-101.4\left(c 0.85, \mathrm{CHCl}_{3}\right)$. This is a known compound. ${ }^{6}$ Enantiomeric excess was determined by chiral HPLC analysis of the 3,5dinitrobenzoate ester: Chiracel AD-H column; 95:5 hexane:isopropanol; $1.0 \mathrm{~mL} / \mathrm{min}, 254 \mathrm{~nm}$.
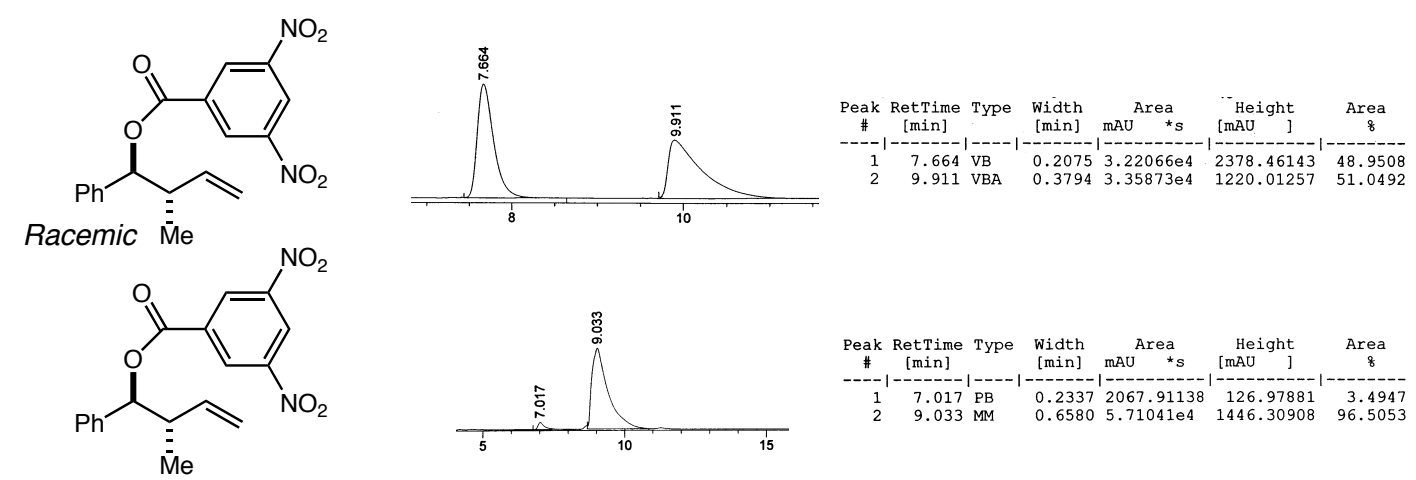

Table 2, Entry 6

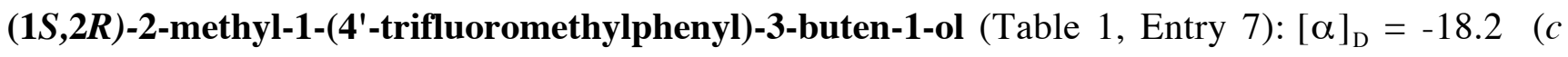
$0.565, \mathrm{CH}_{2} \mathrm{Cl}_{2}$ ). This is a known compound. ${ }^{7}$ Enantiomeric excess was determined by chiral GC: Chiraldex GTA column; $100{ }^{\circ} \mathrm{C} ; 2.0 \mathrm{~mL} / \mathrm{min}$.

(6) Roush, W. R.; Ando, K.; Powers, D. B.; Palkowitz, A. D.; Halterman, R. L.; J. Am. Chem. Soc. 1990, 112, 6339-6348.

(7) Denmark, S. E.; Fu, J.; J. Am. Chem. Soc. 2001, 123, 9488-9489. 

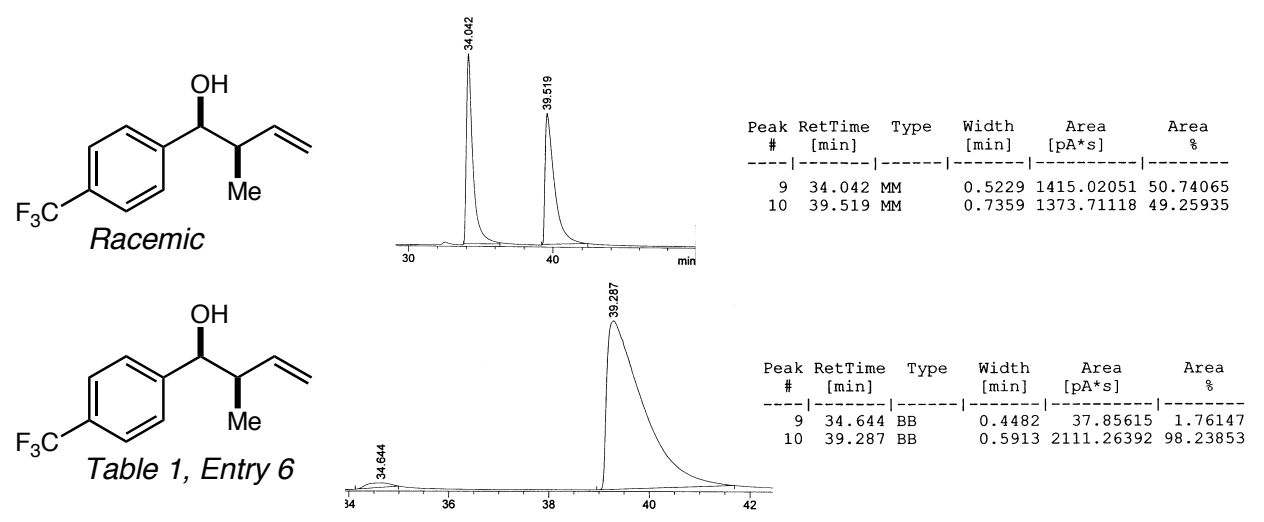

(1S,2S)-2-methyl-1-(4'-trifluoromethylphenyl)-3-buten-1-ol (Table 2, Entry 7): $[\square]_{\mathrm{D}}=-77.1^{\circ}(c$ 1.230, $\mathrm{CHCl}_{3}$ ). This is a known compound. ${ }^{8}$ Enantiomeric excess was determined by chiral GC: Chiraldex GTA column; $100{ }^{\circ} \mathrm{C} ; 2.0 \mathrm{~mL} / \mathrm{min}$.
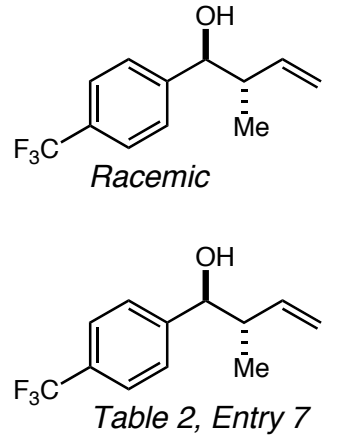
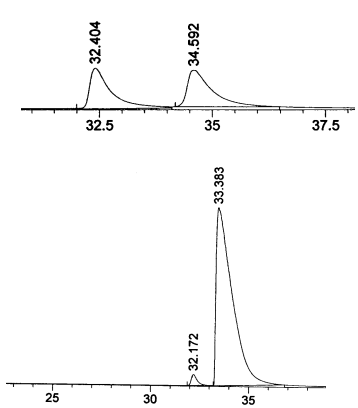
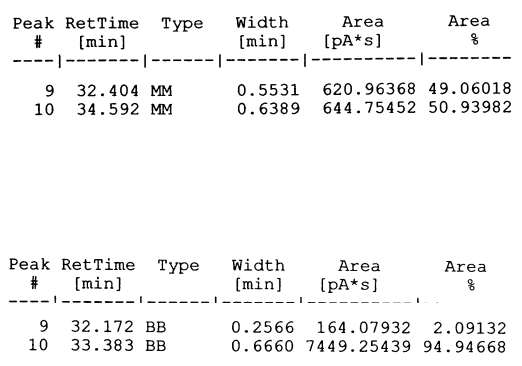

(3S,4R)-4-Methyl-1-phenyl-hexa-1,5-dien-3-ol (Table 1, Entry 8): [ $]_{\mathrm{D}}=-7.1^{\circ}\left(\mathrm{c} 0.59, \mathrm{CH}_{2} \mathrm{Cl}_{2}\right)$. (3S,4S)-4-Methyl-1-phenyl-hexa-1,5-dien-3-ol (Table 2, Entry 8): $[\square]_{\mathrm{D}}=+18.6^{\circ}\left(c 0.955, \mathrm{Et}_{2} \mathrm{O}\right)$. These are known compounds. ${ }^{7}$ Enantiomeric excess was determined by chiral HPLC: Chiracel OD column; hexane to $98: 2$ hexane:isopropanol gradient over $1 \mathrm{~h} ; 1.0 \mathrm{~mL} / \mathrm{min}, 254 \mathrm{~nm}$. The assay was developed using a 1.5:1 syn:anti mixture of racemic diastereomers.

(8) Malkov, A.; Dufková, L.; Farrugia, L.; Kocovsky, P. Angew. Chem. Int. Ed. 2003, 42, 3674-3677. 


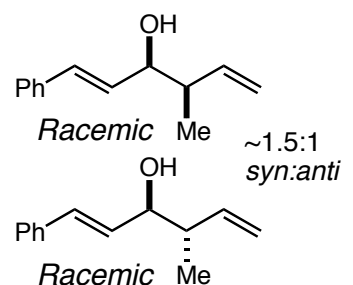<smiles>C=CC(O)C(O)C=Cc1ccccc1</smiles>

Table 1, Entry 8<smiles>C=C[C@H](O)C(O)/C=C/c1ccccc1</smiles>

Table 2, Entry 8
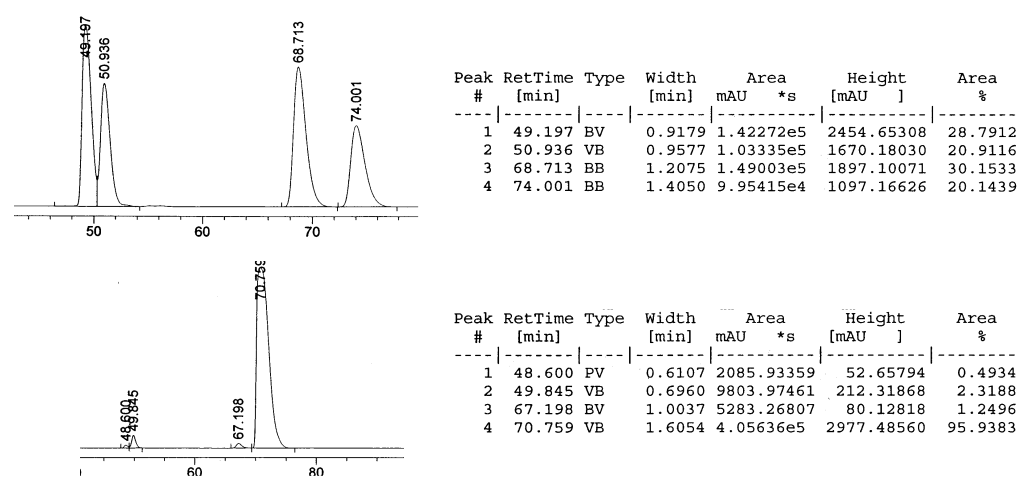

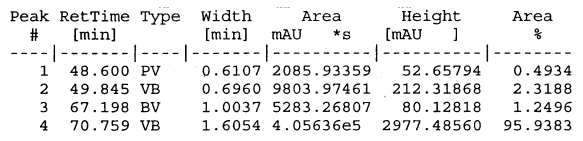

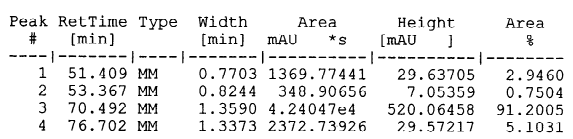

${ }^{1} \mathrm{H}$ NMR Spectrum of $(R, R)-2\left(300 \mathrm{MHz}, \mathrm{CDCl}_{3}\right)$ :

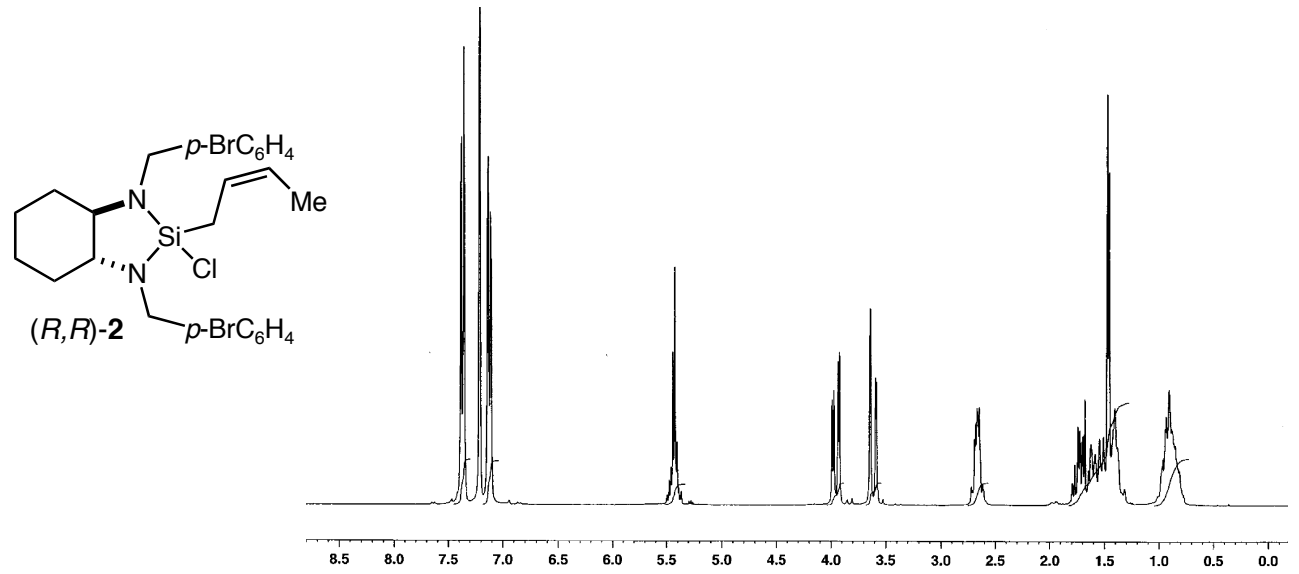

${ }^{1} \mathrm{H}$ NMR Spectrum of $(R, R)-3\left(300 \mathrm{MHz}, \mathrm{CDCl}_{3}\right)$ :

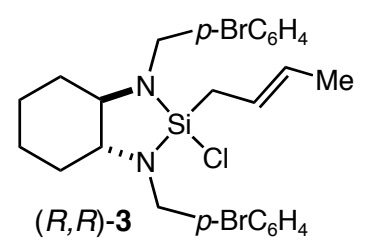<smiles>C1CCCCC1</smiles>

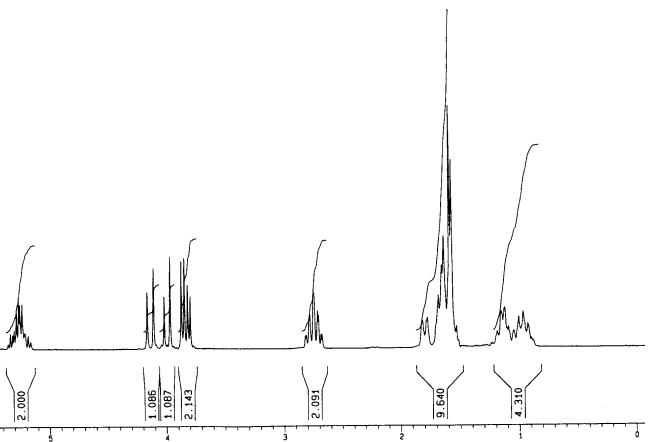


${ }^{1} \mathrm{H}$ NMR Spectrum of (3R,4R)-3,6-dimethyl-1-hepten-4-ol (Table 1, entry 2) (300 MHz, $\left.\mathrm{CDCl}_{3}\right)$ :

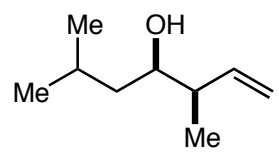

Table 1, Entry 2

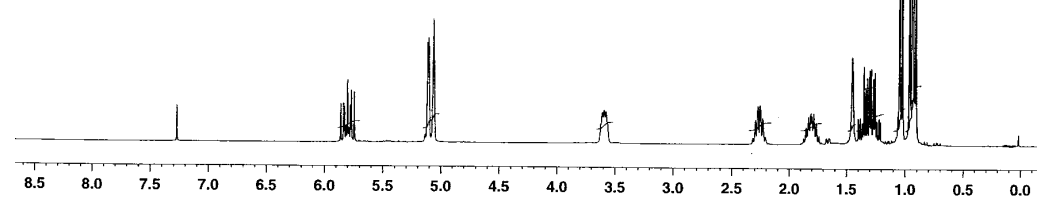

${ }^{1} \mathrm{H}$ NMR Spectrum of (3S,4R)-3,6-dimethyl-1-hepten-4-ol (Table 2, entry 2) (300 MHz, $\left.\mathrm{CDCl}_{3}\right)$ :

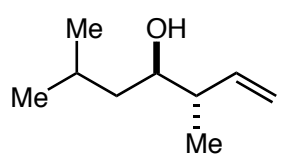

Table 2, Entry 2

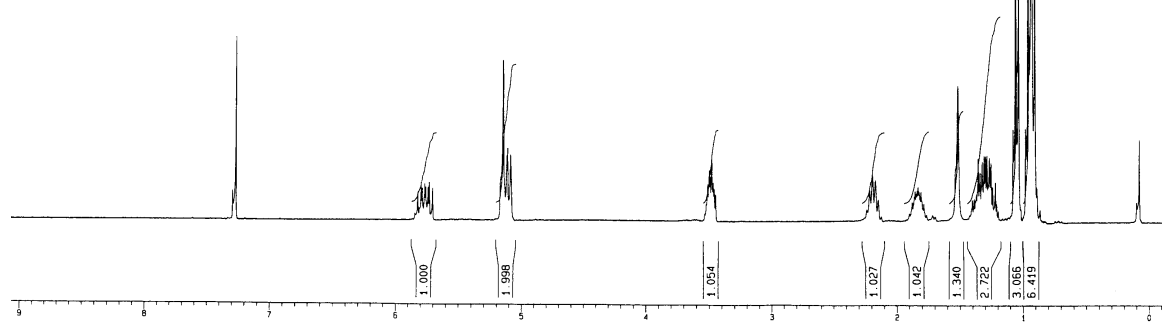


${ }^{1} \mathrm{H}$ NMR Spectrum of (2R,3R)-1-benzyloxy-3-methyl-4-penten-2-ol (Table 1, entry 4) (300 MHz, $\left.\mathrm{CDCl}_{3}\right)$ :

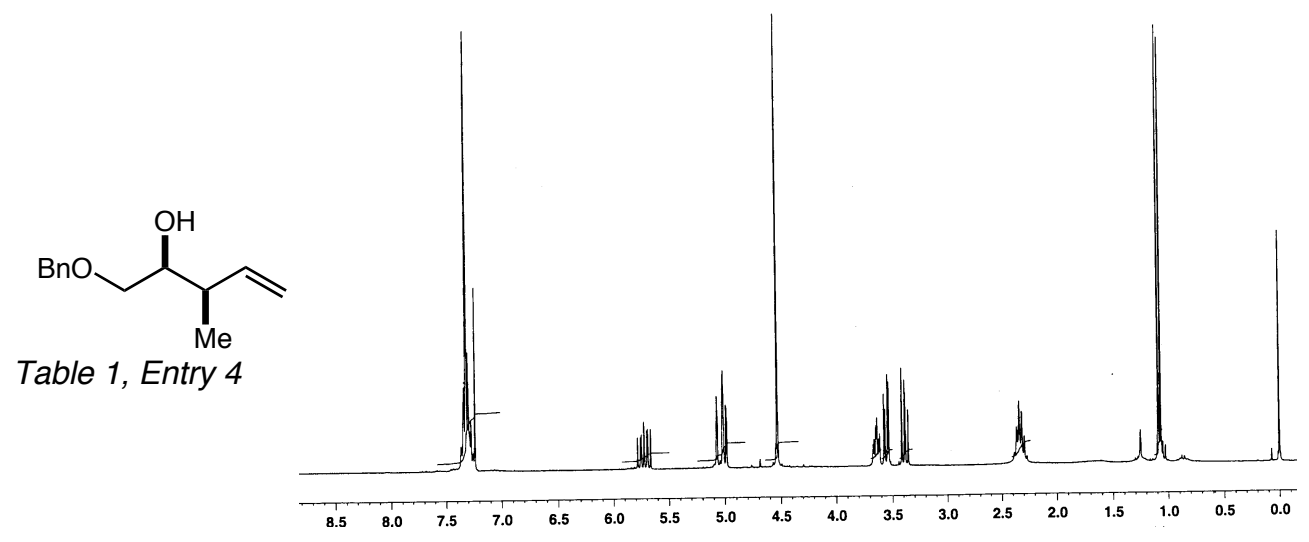

${ }^{1}$ H NMR Spectrum of (3R,4R)-1-(4'-methoxy-benzyloxy)-4-methyl-5-hexen-3-ol (Table 1, entry 5) $\left(300 \mathrm{MHz}, \mathrm{CDCl}_{3}\right)$ :

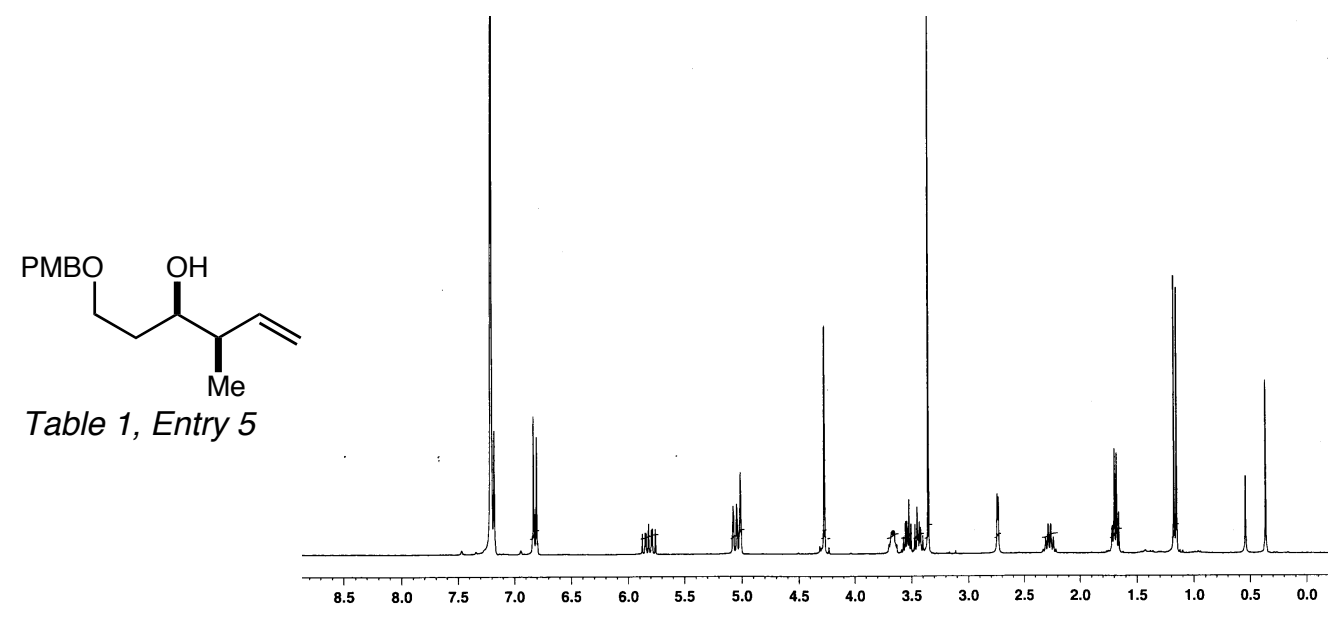

